\title{
Análises de dados longitudinais em bovinos Nelore Mocho por meio de modelos não lineares
}

\author{
Lopes, F.B. ${ }^{\text {; }}$ Magnabosco, C.U. ${ }^{2}$; de Souza, F.M. ${ }^{3}$; de Assis, A.S. ${ }^{3}$ e Brunes, L.C. ${ }^{3}$
}

'FAPEG/Embrapa Cerrados. Planaltinha. DF. Brasil

${ }^{2}$ Embrapa Cerrados/CNPq. Planaltina. DF. Brasil.

${ }^{3}$ Escola de Veterinária de Zootecnia. Universidade Federal de Goiás. Goiânia. Goiás. Brasil.

\section{PalaVRas chaVe adicIONAIS}

Curva de crescimento.

Maturidade.

Melhoramento genético.

Peso assintótico.

\begin{abstract}
RESUMO
Objetivou-se determinar o modelo mais adequado para descrever o crescimento de bovinos Nelore Mocho. Quatro modelos não lineares (Brody, Von Bertalanffy, Gompertz e Logístico), foram ajustados pelo método de Gauss-Newton, utilizando o procedimento NLIN do SAS. Os dados de peso e idade do nascimento à idade adulta de bovinos nascidos entre 2000 e 2013 foram provenientes do rebanho da Empresa Brasileira de Pesquisa Agropecuária - Embrapa Cerrados, situada em Brasília - DF. A qualidade do ajuste dos modelos foi feita por meio do quadrado médio do resíduo, do coeficiente de determinação ajustado, do erro de predição, do desvio médio absoluto dos resíduos, da interpretação biológica dos parâmetros peso assintótico $(A)$ e taxa de maturidade absoluta $(k)$, do gráfico de pesos observados e estimados, da taxa de crescimento instantânea e relativa, bem como da taxa de maturidade absoluta. As estimativas médias para $A$ e $\mathrm{k}$ foram $543,40 \mathrm{~kg}$ e 0,00138\%/dia, $402,00 \mathrm{~kg}$ e 0,00323\%/dia, 378,00 kg e 0,00410\%/dia e 346,00 kg e 0,00657\%/dia, para os Modelos de Brody, Von Bertalanffy, Gompertz e Logístico respectivamente. Dentro do período analisado, o modelo de Brody foi o mais indicado para representar a curva de crescimento de animais da raça Nelore Mocho.
\end{abstract}

\section{Analysis of longitudinal data in Polled Nellore cattle using nonlinear models}

\section{SUMMARY}

This study was carried out to estimate the best different nonlinear models to predict growth curve in Polled Nellore cattle. The models used (Brody, Von Bertalanffy, Gompertz and Logistic models) were fitted by Gauss-Newton method to weight-age data of birth to adult age of Nellore cattle from Brasilia - DF. The adjustment quality was showed by mean square error, adjusted determination coefficient, prediction error, absolute mean deviation residuals, asymptotic weight $(\mathrm{A})$ and maturity rate $(\mathrm{k})$ biological parameters, graphics of observed and estimated weights, of instantaneous and relative growth rate and absolute maturity. The convergence criteria were attained for all nonlinear models. Estimative mean for $\mathrm{A}$ and $\mathrm{k}$ was $543.40 \mathrm{~kg}$ and $0.00138 \% /$ day; $402.00 \mathrm{~kg}$ and $0.00323 \% /$ day; $378.00 \mathrm{~kg}$ and $0.00410 \%$ /day; and $346.00 \mathrm{~kg}$ and $0.00657 \%$ /day for Brody, Von Bertalanffy, Gompertz and Logistic nonlinear models, respectively. Data adjustment for weight-age of the animal provides information describing the growth curve and the future prognostic for animals managed under the same environmental situation. In the period analyzed, Brody nonlinear model was indicated to estimate the body weight of Polled Nellore.

INFORMACIÓN

Cronología del artículo.

Recibido/Received: 06.07.2015

Aceptado/Accepted: 20.01 .2016

On-line: 11.06 .2016

Correspondencia a los autores/Contact e-mail:

ludmillabrunes@hotmail.com

\section{INTRODUÇÃO}

O crescimento dos animais está relacionado com a capacidade de ganho de massa corporal, sendo influenciado pela alimentação, condições climáticas, sanidade e genética. $\mathrm{O}$ conhecimento desse processo é muito importante para os sistemas de produção de carne, pois é possível identificar fases de vida do animal que descrevem diferentes velocidades de crescimento. Sa- bendo disso, é possível adotar estratégias de manejo nutricional para cada etapa de vida do animal ou de seleção genética, focando no melhoramento de características (pesos) que apresentam maior importância no crescimento animal, de forma a maximizar a produtividade e os lucros do sistema (Lopes et al., 2011; Malhado et al., 2009). O crescimento dos bovinos é um processo complexo, pois é influenciado por vários fatores, e é variável nos indivíduos, seguindo, contudo, um padrão 
bem definido em uma população de animais de mesma genética (Brody, 1945; Arango and Vleck, 2002). Este processo pode ser analisado eficientemente por meio de estudos de curvas de crescimento, que relaciona o peso do indivíduo e sua respectiva idade. Para isso, é necessário um conjunto de pesagens do animal, desde o nascimento até a maturidade (Oliveira, 1995).

O estudo de curvas de crescimento consiste em sintetizar informações de todo o período de vida dos animais, por meio de ajustes de funções não lineares em um pequeno conjunto de parâmetros matemáticos com interpretação biológica (Lopes et al., 2011). A utilização dessa tecnologia evita os inconvenientes de interpretação independente de um grande número de medidas isoladas, que estão sujeitas a efeitos ambientais temporários não identificáveis (Fitzhugh Jr., 1976; Forni, 2007). Um exemplo disso, é a seleção de animais mais pesados em determinada idade com intuito de obter indivíduos mais precoces, quando na verdade essa informação não é necessariamente plausível, pois o peso está relacionado ao tamanho corporal do animal, e quanto maior o animal, maiores são as demandas nutricionais, mais tempo requererá para atingir a maturidade.

Por meio do estudo de curvas de crescimento é possível obter diferentes tipos de parâmetros que podem ser analisados para melhor entender o comportamento do crescimento animal. Um deles é a taxa de maturidade (k), uma função que envolve a máxima taxa de crescimento e o peso adulto do animal. Quanto maior esse valor, mais precoce será o animal em termos de crescimento, pois atingirá o peso adulto em menor tempo. A taxa de maturidade tem demonstrado herdabilidade moderada, e isso significa que a seleção para esse parâmetro pode ser mais eficaz quando o objetivo é obter animais precoces, do que a seleção para uma isolada característica de peso (Santoro et al., 2005).

Segundo Malhado et al. (2009) os modelos mais utilizados para descrever o crescimento dos animais são as funções Brody, Von Bertalanffy, Richards, Gompertz e Logístico. Para Silva et al. (2004) o modelo de Gompertz foi o que melhor se ajustou aos dados, enquanto Silva et al. (2011a) relataram que Brody foi o mais adequado. Segundos estes autores o ajuste do modelo pode variar de acordo com a raça, ambiente, idade do animal nas últimas pesagens e do modelo propriamente dito.

A escolha do método mais adequado pode ser feita por meio da avaliação da soma de quadrados residuais, desvios de regressão, coeficiente de determinação $\left(R^{2}\right)$, porcentagem e dificuldade de convergência, comportamento das curvas, avaliação dos parâmetros por comparação e avaliação gráfica das curvas (Fitzhugh Jr, 1976; Brown et al., 1976; Perotto et al., 1992).

Vários pesquisadores utilizam modelos não lineares em estudos de crescimento de bovinos (Perotto et al., 1992; Mazzini et al., 2003; Souza et al., 2010; Lopes et al., 2011). Entretanto, os estudos relativos às curvas de crescimento exclusivamente em bovinos Nelore de variedade mocha são incipientes. Ademais, existem poucos estudos que avaliam os pesos durante todo o ciclo de produção de zebuínos, desde o nascimento até a idade adulta e, assim, não há uma descrição global do crescimento que possa ser considerado como padrão médio de crescimento para zebuínos (Oliveira et al., 2000), incluindo bovinos Nelore Mocho. Assim, objetivou-se analisar e determinar o modelo não linear que melhor se ajusta aos dados de crescimento para peso e idade de um rebanho de bovinos da raça Nelore Mocho.

\section{MATERIAL E MÉTODOS}

Foram utilizadas pesagens de animais manejados em sistema extensivo de criação. Os dados analisados correspondem as pesagens de 217 matrizes nascidas entre 2000 e 2013, provenientes de rebanhos da Empresa Brasileira de Pesquisa Agropecuária - Embrapa Cerrados, situada em Brasília - DF.

Para análise dos modelos não lineares, os registros de pesagens das fêmeas foram mensurados do nascimento à idade adulta. As pesagens foram realizadas em intervalos médios de três meses, sendo coletadas cerca de nove informações de cada animal. Com base nestes registros, realizaram-se análises dos parâmetros da curva de crescimento como peso assintótico (A), taxa de maturidade $(\mathrm{k})$, taxa de crescimento instantâneo (TCI), taxa de crescimento instantâneo relativo (TCIR) e taxa de maturidade absoluta (TMA).

Os modelos utilizados para ajustar os pesos às idades de cada animal e suas respectivas parametrizações foram: Brody (I), Von Bertalanffy (II), Gompertz (III) e Logístico (IV).

$$
\begin{aligned}
& y_{t}=A\left(1-B \exp ^{-k t}\right) \\
& y_{t}=A\left(1-B \exp ^{-k t}\right)^{3} \\
& y_{t}=A \exp \left(-B \exp ^{-k t}\right) \\
& y_{t}=A /\left(1+B \exp ^{-k t}\right)
\end{aligned}
$$

Em que $y_{t}$ é o peso corporal aos t meses de idade; $A$ é a estimativa do peso assintótico; B é uma constante de integração; e, k é a taxa de maturação.

Estes modelos foram ajustados aos dados de peso e idade de animais da Raça Nelore Mocho, por meio do procedimento NLIN do SAS $®$ (2002). As estimativas dos parâmetros dos modelos foram obtidas por processo iterativo de Gauss-Newton, adotando-se o critério de convergência de $10^{-8}$. Para a comparação dos modelos não lineares, quanto ao ajustamento aos dados de peso e idade, foram utilizados os critérios quadrado médio do resíduo, coeficiente de determinação, erro médio de predição e desvio médio absoluto dos resíduos.

Para descrever o crescimento dos animais, foram analisadas taxa de crescimento instantânea (TCI), a qual estima o incremento no peso do animal para cada unidade de tempo t; taxa de crescimento instantânea relativa (TCIR), que estima a taxa de crescimento instantânea em relação ao peso do indivíduo no tempo t; e taxa de maturidade absoluta (TMA), razão entre a taxa de crescimento instantânea e o peso assintótico A, que representa a taxa de crescimento instantânea em relação ao tamanho global. 
Tabela I. Peso assintótico (A), taxa de maturidade (k), estimativa de correlação entre A e $k$ ( $\left.\mathrm{r}_{\mathrm{Ak}}\right)$, número de interações para convergir (NIC), quadrado médio do resíduo (QMR), coeficiente de determinação ( $\left.{ }^{2}\right)$, erro médio de predição (EMP) e desvio médio absoluto (DMA), em fềmeas da raça Nelore (Asymptotic weight (A), maturity rate $(k)$, estimated correlation between $A$ and $k\left(r_{A k}\right)$, number of interactions to convergence (NIC), mean square error (QMR), coefficient of determination $\left(R^{2}\right)$, mean error of prediction (EMP), absolute deviation mean (DMA), in Nellore cattle females).

\begin{tabular}{|c|c|c|c|c|c|c|c|c|}
\hline Modelo & $\begin{array}{c}\text { A } \\
(\mathrm{kg})\end{array}$ & $\begin{array}{c}\mathrm{k} \\
(\% / \mathrm{dia})\end{array}$ & $r_{A K}$ & NIC & QMR & $\mathrm{R}^{2}$ & EMP & $\begin{array}{l}\text { DMA } \\
(\mathrm{kg})\end{array}$ \\
\hline Brody & 522 & 0,00148 & $-0,78$ & 5 & $1018,61^{\mathrm{c}}$ & $0,98^{A}$ & $0,94^{\mathrm{A}}$ & $18,5^{\mathrm{D}}$ \\
\hline Von Bertalanffy & 502 & 0,00243 & $-0,62$ & 11 & $1235,35^{\mathrm{B}}$ & $0,97^{\mathrm{B}}$ & $0,53^{A}$ & $22,88^{\circ}$ \\
\hline Gompertz & 495 & 0,00299 & $-0,54$ & 11 & $1391,03^{\mathrm{AB}}$ & $0,96^{\mathrm{C}}$ & $-0,15^{A}$ & $25,72^{\mathrm{B}}$ \\
\hline Logístico & 490 & 0,00354 & $-0,51$ & 12 & $1533,62^{\mathrm{A}}$ & $0,95^{\mathrm{D}}$ & $-0,23^{A}$ & $27,71^{\mathrm{A}}$ \\
\hline
\end{tabular}

${ }^{A B C}$ Letras maiúsculas distintas na coluna indicam diferença estatisticamente significativa $(p<0,01)$ pelo teste de Duncan.

Foram realizadas análises de variância dos parâmetros de ajustes dos modelos por meio do procedimento GLM, utilizando o seguinte modelo matemático:

$$
y=\mu+\mathrm{M} i+\varepsilon
$$

Em que $y$ é o parâmetro de ajuste de cada modelo $\mathrm{M} i$; e $\varepsilon$ é o resíduo, normalmente distribuído, com média zero e variância $\sigma_{\varepsilon}^{2}$.

As médias foram comparadas por meio do teste de Duncan $(p<0,05)$. Para organizar as informações sobre os modelos não lineares e pesos observados, com a finalidade de formação dos grupos similares, foi utilizado o procedimento CLUSTER. O método adotado para a definição dos clusters foi o método da mínima variância (Ward), no qual um grupo é reunido a outro grupo, se essa união proporcionar o menor aumento da variância intragrupo. Com a adoção desse método, a variância intragrupo é calculada para todas as possibilidades de aglomeração, optando-se pelo arranjo que proporcione a menor variância. A eficiência do método hierárquico de Wald foi testada por meio dos critérios do pseudo-Fe pseudo-t² (Gonçalves et al., 2008). Os gráficos foram criados por meio dos procedimentos GPLOT e TREE. Todas as análises, univariadas e multivariadas, foram realizadas utilizando-se o programa computacional Statistical Analysis System (SAS, 2002).

\section{RESULTADOS E DISCUSSÃO}

O peso médio à idade adulta foi de $555 \pm 71 \mathrm{~kg}$ e em todos os modelos o peso assintótico foi subestimado em relação ao peso médio observado na respectiva idade. Apenas o modelo de Brody se aproximou do peso médio observado com estimativa de $522 \mathrm{~kg}$ (tabela I).

A taxa de maturidade, representada pelo parâmetro $k$ das curvas de crescimento, indica a precocidade dos animais em estudo. Observou-se que à medida que o peso assintótico aumentou houve diminuição da taxa de maturidade. Isto pôde ser observado por meio das estimativas de correlação entre o peso assintótico e a taxa de maturidade, as quais apresentaram estimativa média de correlação em torno de -0,61 (tabela I), corroborando com resultados obtidos por vários autores (McManus et al., 2003; Freitas, 2005; Malhado et al., 2009; Lopes et al., 2011), os quais também evidenciaram comportamento antagônico entre $A$ e $k$, ou seja, animais mais pesados em idade adulta possuem menor velocidade de crescimento. Isso é justificado

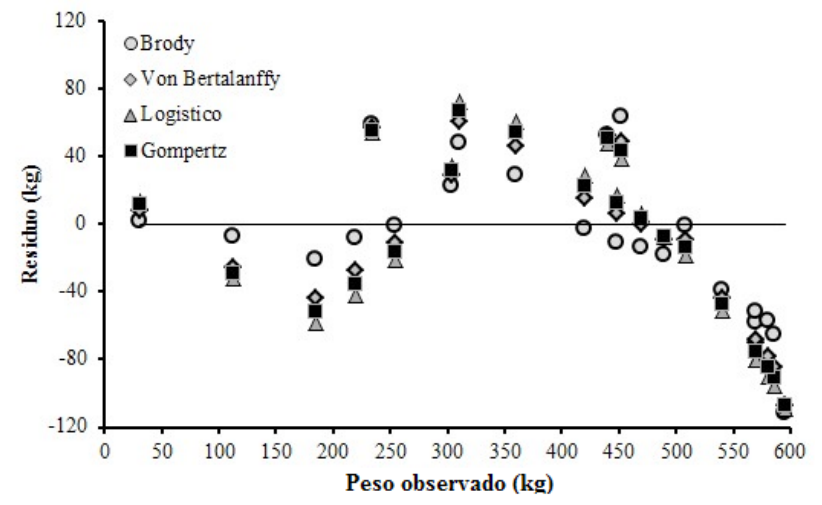

Figura 1. Regressão dos resíduos dos pesos preditos pelos modelos de Brody, Von Bertalanffy, Gompertz e Logístico em função dos pesos observados de fêmeas da raça Nelore (Regression of residuals predicted weights for Brody, Von Bertalanffy, Gompertz and Logistic models in function of observed weights to Nellore cattle females).

pela relação existente entre peso e tamanho corporal dos animais, pois maiores pesos são apresentados por animais com maiores medidas corporais e vice-versa. Assim, indivíduos com maior tamanho corporal adulto apresentam maiores requerimentos nutricionais de manutenção, sendo mais tardios, por isso apresentarão menor velocidade de crescimento.

Dentre todos os modelos analisados, o modelo Brody apresentou menor variância residual (figura 1), indicada pela pequena estimativa do desvio médio absoluto (DMA) e do quadrado médio do resíduo (QMR), como pode ser visto na tabela I. O melhor resultado para o coeficiente de determinação também foi observado para o modelo de Brody $(0,98)$, demonstrando ser o modelo que melhor se ajustou aos dados, pois indicou maior associação entre pesos observados e estimados (figura 2).

No estudo de Silva et al. (2011a) foi demonstrado que, pesos de vacas de diferentes grupos genéticos, também evidenciaram resultados semelhantes e concluíram que os modelos de Brody e o de Von Bertalanffy apresentaram-se adequados para ajustar as curvas de crescimento, do nascimento até a idade adulta.

Embora todos os modelos tenham convergido e, com base nos parâmetros de ajustes de cada um deles (quadrado médio do resíduo, coeficiente de determinação, erro médio de predição e desvio médio abso- 


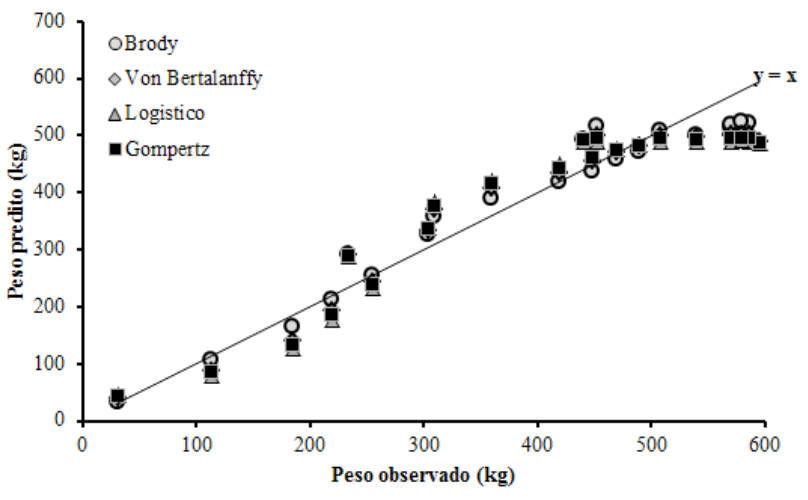

Figura 2. Regressão dos pesos preditos pelos modelos de Brody, Von Bertalanffy, Gompertz e Logístico em função dos pesos observados de fêmeas da raça Nelore (Regression of predicted weights for Brody, Von Bertalanffy, Gompertz and Logistic models in function of observed weights to Nellore cattle females).

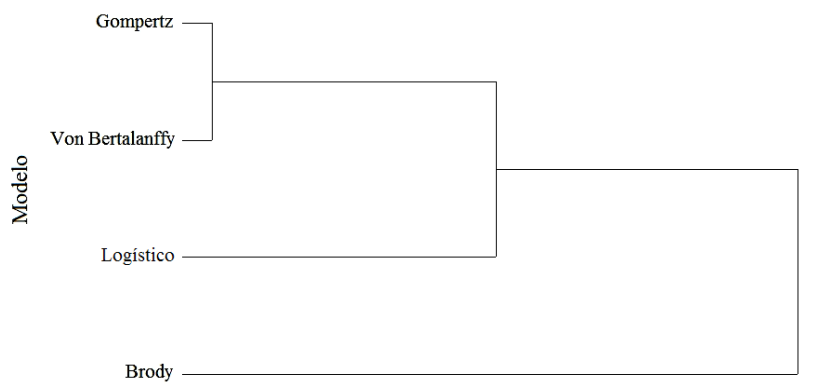

$\begin{array}{llllllllllllll}0,00 & 0,05 & 0,10 & 0,15 & 0,20 & 0,25 & 0,30 & 0,35 & 0,40 & 0,45 & 0,50 & 0,55 & 0,60 & 0,65\end{array}$

Figura 3. Dendrograma dos parâmetros de ajustes (quadrado médio do resíduo, coeficiente de determinação, erro médio de predição e desvio médio absoluto), dos modelos de Brody, Von Bertalanffy, Gompertz e Logístico (Dendrogram of the fitted parameters (residual mean square, coeficiente of determination, prediction mean error and mean absolute deviation) using the Brody, Von Bertalanffy, Gompertz and Logistic models).

luto), foi possível discriminá-los por meio de análises de Cluster (figura 3). Os modelos de Gompertz e Von Bertalanffy foram agrupados no mesmo cluster. Brody e Logístico agruparam-se distintamente. O modelo Logístico apresentou resultados menos satisfatórios, enquanto que o modelo de Brody apresentou os melhores ajustes. Logo, sugere-se a utilização deste para descrever o comportamento de crescimento e desenvolvimento dos animais do rebanho analisado. Este resultado corroborou com o estudo de Silva et al. (2011b), que analisando diferentes modelos não lineares para descrever o crescimento de bovinos Nelore, observaram que o modelo de Brody foi o que melhor se ajustou ao crescimento dos animais dessa raça.

Todos os modelos superestimaram o peso médio ao nascimento e subestimaram o peso médio à idade de 631 dias. Dentre todos os modelos o que melhor ajustou os pesos às respectivas idades foi o modelo de Brody (tabela II). Esses resultados vão de encontro às observações de Forni (2007), o qual afirma que a equação de Brody é a mais utilizada para descrever dados de crescimento de bovinos de corte, em resultado
Tabela II. Médias para idade, pesos observados e preditos pelos modelos de Brody, Von Bertalanffy, Gompertz e Logístico, de acordo com a idade das fêmeas da raça Nelore (Age average, observed and predicted weights using Brody, Von Bertalanffy, Gompertz and Logistic models in accordance to Nellore cattle females age).

\begin{tabular}{lrrrrr}
\hline \multirow{2}{*}{$\begin{array}{l}\text { Idade média } \\
\text { (dias) }\end{array}$} & \multirow{2}{*}{$\begin{array}{c}\text { Pesos } \\
(\mathrm{kg})\end{array}$} & \multicolumn{5}{c}{ Modelo $(\mathrm{kg})$} \\
\cline { 3 - 6 } & & \multicolumn{1}{c}{$\mathrm{B}$} & \multicolumn{1}{c}{ VB } & \multicolumn{1}{c}{$\mathrm{G}$} & \multicolumn{1}{c}{$\mathrm{L}$} \\
\hline Nascimento & 31,75 & 38,09 & 46,15 & 49,99 & 59,47 \\
80 & 91,11 & 90,65 & 88,10 & 88,01 & 89,97 \\
161 & 150,00 & 138,88 & 134,86 & 133,17 & 129,88 \\
241 & 190,58 & 180,96 & 179,20 & 177,95 & 174,08 \\
327 & 209,99 & 220,86 & 221,70 & 221,61 & 220,50 \\
420 & 246,88 & 259,66 & 261,49 & 262,09 & 263,47 \\
514 & 290,52 & 294,31 & 294,47 & 294,61 & 295,72 \\
586 & 330,87 & 317,87 & 314,83 & 313,79 & 312,49 \\
631 & 355,34 & 331,41 & 325,85 & 323,86 & 320,58 \\
\hline
\end{tabular}

$\mathrm{B}=$ Brody; $\mathrm{VB}=$ Von Bertalanffy; $\mathrm{G}=\mathrm{Gompertz} ; \mathrm{L}=$ Logístico.

da facilidade de convergência dos parâmetros, mesmo na falta de dados, pelo menor esforço computacional e qualidade de ajuste.

Considerando o peso médio observado de 355,34 $\mathrm{kg}$ à idade média de 21 meses, o modelo que melhor se ajustou, embora também tenha superestimado o peso observado, foi o predito pelo modelo de Brody com média de 331,41 kg. Os modelos de Von Bertalanffy, Gompertz e Logístico também subestimaram o peso assintótico, apresentando estimativas de peso médio de $325,85,323,86$ e $320,58 \mathrm{~kg}$, respectivamente.

A figura 4, desenvolvida com objetivo de avaliar, por meio dos modelos testados, se os pesos preditos estão de acordo com o peso observado, demonstrou que o modelo de Brody foi o mais adequado para descrever a curva de crescimento na raça Nelore Mocho e, dessa forma, pode-se indicar a curva descrita pelo modelo, com os parâmetros encontrados na análise, como um padrão médio de crescimento em fêmeas da raça Nelore Mocho. O modelo de Brody também foi considerado o mais indicado para descrever o crescimento de animais da raça Nelore por Garnero et al. (2005), Perotto et

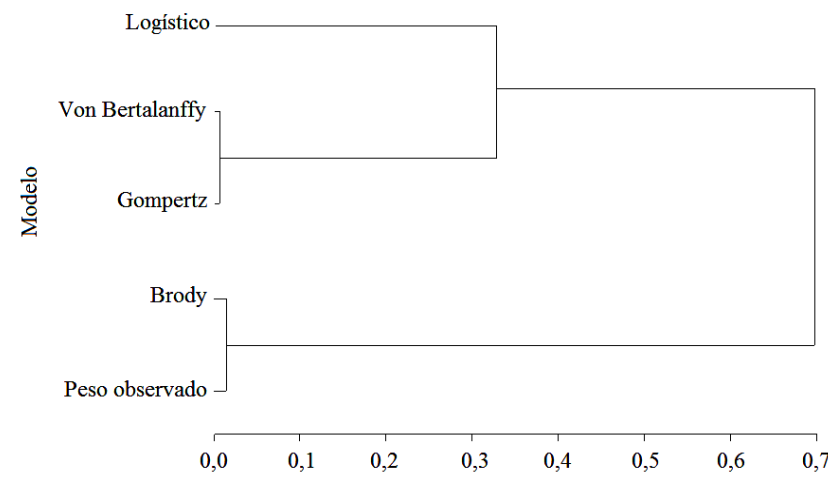

Figura 4. Dendrograma conjunto dos pesos observados e dos pesos preditos por meio dos modelos de Brody, Von Bertalanffy, Gompertz e Logístico (Dendrogram of observed weight together of predicted weights using the Brody, Von Bertalanffy, Gompertz and Logistic models). 


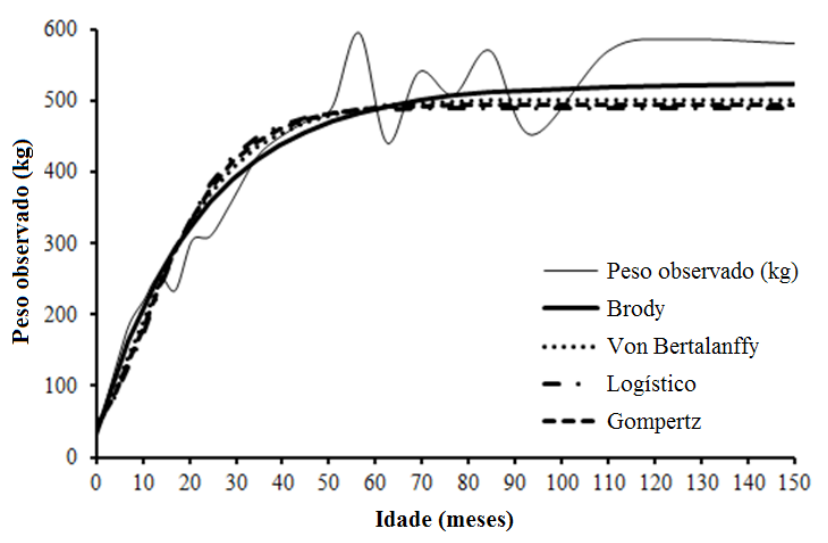

Figura 5. Curvas de crescimento estimadas por meio dos modelos de Brody, Von Bertalanffy, Gompertz, Logístico e dos pesos observados de fêmeas da raça Nelore (Growth curve estimated using observed and predicted weights by Brody, Von Bertalanffy, Gompertz and Logistic models to Nellore cattle females).

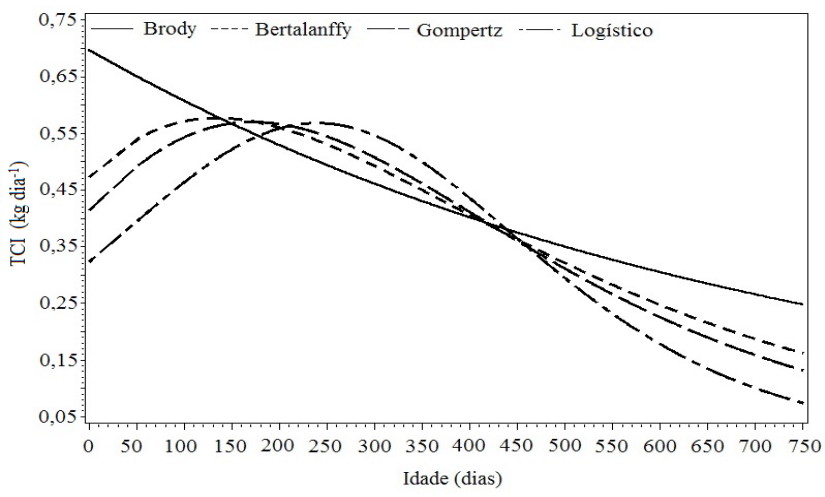

Figura 6. Taxa de crescimento instantâneo (TCI, kg dia-1) obtida por meio dos modelos de Brody, Von Bertalanffy, Gompertz e Logístico para fêmeas da raça Nelore (Instant growth rate (IGR, kg days-1) using the Brody, Von Bertalanffy, Gompertz and Logistic models to Nellore cattle females).

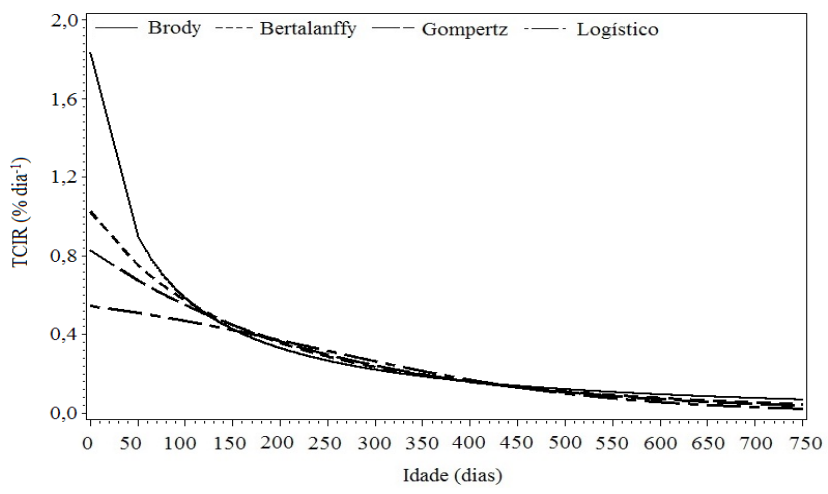

Figura 7. Taxa de crescimento instantâneo relativo (TCIR \% dia-1) obtida por meio dos modelos de Brody, Von Bertalanffy, Gompertz e Logístico para fêmeas da raça Nelore (Relative instant growth rate (RIGR, \% days-1) using the Brody, Von Bertalanffy, Gompertz and Logistic models to Nellore cattle females).

al. (1992) e Oliveira et al. (2000), pois observaram maior similaridade entre os pesos observados e preditos.

Avaliando as curvas de crescimento estimadas por meio dos modelos estudados, observou-se que do nas- cimento aos 50 meses de idade, os modelos proporcionam bom ajuste dos dados. Dos 50 aos 100 meses de idade, o peso real apresenta uma oscilação que não é acompanhada pelas curvas de crescimento preditas. A partir de 100 meses de idade, todos os modelos apresentaram subestimativa dos pesos reais (figura 5).

A figura 6 apresenta a taxa de crescimento instantâneo (TCI) obtida por meio dos modelos avaliados. Observou-se que o modelo de Brody não apresentou ponto de inflexão, em que a TCI mostrou-se sempre decrescente, iniciando com ganho em torno de $700 \mathrm{~g} /$ dia e apresentando ganho médio aos 750 dias de idade de aproximadamente $259 \mathrm{~g} /$ dia. No modelo de Von Bertalanffy a TCI iniciou com ganhos médios de 470 g/dia e foi crescente até atingir o máximo em torno de $560 \mathrm{~g} /$ dia, aproximadamente aos 130 dias de idade, após essa idade, começou a decrescer, chegando a atingir, aos 750 dias de idade, um ganho médio de 170 g/dia. O modelo de Gompertz e Logístico apresentaram à taxa de crescimento instantânea inicial com média de 410 e $310 \mathrm{~g} / \mathrm{dia}$, com ponto de inflexão em torno dos 170 e 250 dias de idade e ganho em peso médio de 560 g/dia. Após esse período, a TCI decresceu até os 750 dias, passando a apresentar menores estimativas de ganho em peso com médias de 130 e 60 g/dia para Gompertz e Logístico, respectivamente.

Nos modelos de Von Bertalanffy, Gompertz e Logístico a taxa de crescimento apresentou aumento do nascimento até atingir o ponto em que o crescimento foi máximo e depois decresceu. Em estudo cujos animais possuem pesagens até a idade adulta, a TCI decresce até atingir valores próximos a zero quando o tamanho máximo do indivíduo é alcançado. Neste estudo, as pesagens foram interrompidas antes que a maturidade fosse atingida, possivelmente, por se tratarem de rebanhos de corte.

Observou-se que aos 750 dias de idade, os animais ainda estavam em desenvolvimento, e apresentaram baixas taxas de crescimento instantâneo. Isto explica a razão pela qual as médias obtidas para o peso assintótico apresentaram estimativas inferiores aos valores normalmente encontrados para o peso adulto de animais da raça Nelore (Garnero et al., 2005; Santoro et al., 2005).

Em todos os modelos a taxa de crescimento instantânea relativa foi decrescente, com melhores resultados para o modelo de Brody (figura 7). A taxa de crescimento instantânea relativa (TCIR) estima a proporção do incremento no peso do animal para cada dia em relação ao seu peso. O modelo de Brody apresentou até os dois meses de idade, as mais elevadas TCIR, seguido pelos modelos de Von Bertalanffy e Gompertz. O modelo Logístico apresentou taxa de crescimento instantânea relativa inferior aos demais modelos, e a partir dos cinco meses de idade, todos os modelos apresentaram decréscimos semelhantes.

A taxa de maturidade absoluta (TMA) indica a proporção do crescimento diário após o nascimento, relativa ao valor assintótico $A$. Neste estudo o parâmetro $A$ representa uma estimativa do peso em torno dos 750 dias de idade, pois os animais não atingiram a maturidade. Para o modelo de Brody, o valor da taxa de maturidade absoluta apresentou estimativa média 


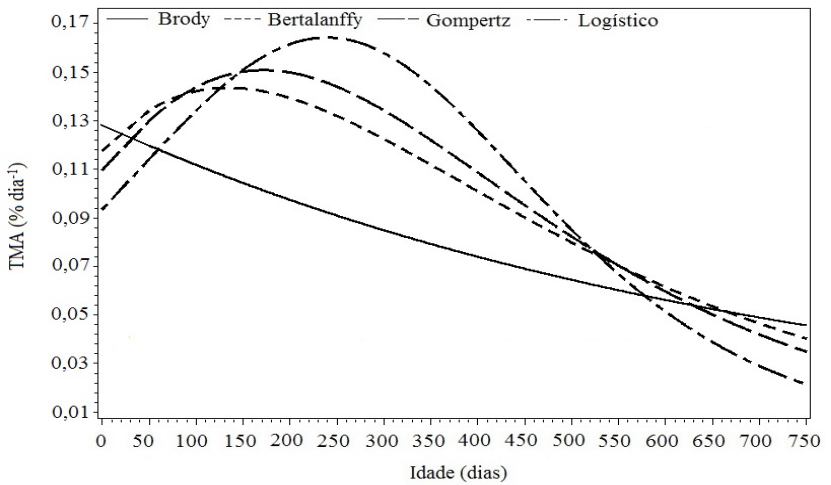

Figura 8. Taxa de maturidade absoluta (TMA \% dia-1) de animais da raça Nelore obtida por meio dos modelos de Brody, Von Bertalanffy, Gompertz e Logístico de fêmeas da raça Nelore (Absolute maturity rate (AMR, \% days-1) in Nellore cattle using the Brody, Von Bertalanffy, Gompertz and Logistic models to Nellore cattle females).

ao nascimento de $0,13 \%$ com constante decréscimo ao longo do tempo. Os modelos de Von Bertalanffy, Logístico e de Gompertz apresentaram taxa de maturidade absoluta de 0,12, 0,11 e 0,10\%, ao nascimento, respectivamente, e apresentaram estimativas máximas de $0,13,0,14$ e $0,15 \%$, aos 120, 175 e 250 dias de idade, respectivamente (figura 8 ).

Constatou-se acentuado decréscimo da taxa de crescimento absoluto na fase pós-desmama, para os modelos de Von Bertalanffy, Gompertz e Logístico, indicando que estratégias nutricionais precisam ser implantadas, com o objetivo de amenizar o pequeno ganho em peso nessa fase, corroborando com resultados observados por Lopes et al. (2011), os quais analisaram nove pesagens de bovinos Nelore Padrão, criados a pasto na região Norte do Brasil. Já em relação a taxa de maturidade absoluta obtida por meio da curva de crescimento de Brody foi observado tendência completamente decrescente.

O conhecimento das estimativas dos parâmetros da curva de crescimento é importante para o controle do rebanho, pois descrevem o crescimento dos animais em função da idade e possuem interpretação biológica (Fitzhugh Jr., 1976, Forni et al., 2007; Forni et al., 2009). Quando se obtém a diferenciação da variável dependente $\mathrm{y}_{\mathrm{t}}$, em função da idade $\mathrm{t}$, novas características relacionadas ao crescimento, em adição ao peso estimado, podem ser investigadas, possibilitando a compreensão de forma simultânea, do processo de crescimento para adoção de técnicas de manejo adequadas a cada fase de vida do animal (Lopes et al., 2011).

Com isso, a utilização das curvas de crescimento pode elucidar fatores que influenciam o crescimento dos animais em determinada idade, evidenciando algum tipo de erro de manejo, passível de ser corrigido. A partir das curvas de crescimento é possivel obter prognósticos futuros para animais do mesmo grupo racial sob a mesma situação de ambiente. Assim, os modelos não lineares, utilizados para descrever o crescimento animal, tanto para fins de exigência nutricional quanto para seleção genética é de extrema importância (Tedeschi et al., 2000).

\section{CONCLUSÕES}

Embora todas as curvas de crescimento estudadas tenham convergido, o modelo Brody apresentou-se como o mais indicado para representar a curva de crescimento média e também para ser utilizado em estudos do crescimento de bovinos da raça Nelore Mocho. As taxas de crescimentos observadas indicaram que estratégias de manejo nutricional precisam ser implementadas de forma a melhorar o ganho em peso de bovinos Nelore Mocho, principalmente após o desmame, fase em que há acentuada redução do crescimento e desenvolvimento do animal.

\section{AGRADECIMENTOS}

À Empresa Guaporé Pecuária (Marca OB) pela concessão dos dados para o desenvolvimento deste trabalho.

\section{BIBLIOGRAFIA}

Arango, J.L. and Vleck, D.V. 2002. Size of beef cows: early ideas, new developments. Genet Mol Res, 1: 51-63.

Brody, S. 1945. Bioenergetics and growth with special reference to the energetic efficiency complex in domestic animals. Reinhold Publ. New York. 1023 pp.

Brown, J.E.; Fitzhugh Jr., H.A. and Cartwright, T.C. 1976. A comparison of nonlinear models for describing weight-age relationships in cattle. J Anim Sci, 42: 810-818.

Fitzhugh Jr, H.A. 1976. Analysis of growth curves and strategies for altering their shape. J Anim Sci, 42: 1036-1051.

Forni, S.; Piles, M.; Blasco, A.; Varona, L.; Oliveira, H.N.; Lôbo, R.B. and Albuquerque, L.G. 2007. Analysis of beef cattle longitudinal data applying a nonlinear model. J Anim Sci, 85: 3189-3197.

Forni, S.; Piles, M.; Blasco, A.; Varona, L.; Oliveira, H.N.; Lôbo, R.B. and Albuquerque, L.G. 2009. Comparison of different nonlinear functions to describe Nellore cattle growth. J Anim Sci, 87:496-506.

Freitas, A.R. 2005. Curvas de crescimento na produção animal. Rev Bras Zootecn, 34: 786-795.

Gonçalves, L.S.; Rodrigues, R.; Amaral, A.T.J.; Karasawa, M. and Sudré, C.P. 2008. Comparison of multivariate statistical algorithms to cluster tomato heirloom accessions. GenetMol Res, 7: 1289-1297.

Garnero, A.D.V.; Marcondes, C.R.; Bezerra, L.A.F.; Oliveira, H.N. e Lôbo, R.B. 2005. Parâmetros genéticos da taxa de maturação e do peso assintótico de fêmeas da raça Nelore. Arq Bras Med Vet Zootec, 57: 652-662.

Lopes, F.B.; Silva, M.C.; Marques, E.G. e McManus, C. 2011 . Ajustes de curvas de crescimento em bovinos Nelore da região Norte do Brasil. Rev Bras Saúde Prod Anim, 12: 607-617.

Lopes, F.B.; Silva, M.C.; Marques, E.G. e McManus, C. 2012. Analysis of longitudinal data of beef cattle raised on pasture from northern Brazil using nonlinear models. Trop Anim Health Prod, 41: 1945-1951.

Malhado, C.H.M.; Ramos, A.A.; Carneiro, P.L.S.; Azevedo, D.M.M.R.; Melo, P.R.A.R.; Pereira, D.G.; Souza, J.L. e Martins Filho, R. 2009. Modelos não lineares utilizados para descrever o crescimento de bovinos da raça Nelore no estado da Bahia: 1. Efeito ambiental. Rev Bras Saúde Prod Anim, 10: 821-829.

Mazzini, A.R.S.; Muniz, J.A.; de Aquino, L.H. e Silva, F.F. 2003. Análise da curva de crescimento de machos Hereford. Cienc Agrotec, 27:1105-1112.

McManus, C.; Evangelista, L.A.C.; Fernandes, R.M.; Miranda, F.E.; Moreno-Bernal, F.E. e Santos, N.R. 2003. Curvas de crescimento de ovinos Bergamácia criados no Distrito Federal. Rev Bras Zootecn, 32: 1207-1212

Oliveira, H.N. 1995. Analise genético-quantitativo da curva do crescimento de fêmeas da raça Guzerá. Tese (Doutorado em Ciências). Faculdade de Medicina Veterinária. USP. Ribeirão Preto. Brasil. 73 pp. 
Oliveira, H.N.; Lôbo, R.B. e Pereira, C.S. 2000. Comparação de modelos não-lineares para descrever o crescimento de fêmeas da raça Guzerá. Pesq Agropec Bras, 35: 1843-1851.

Perotto, D.; Cue, R.I. and Lee, A.J. 1992. Comparison of nonlinear functions for describing the growth curve of three genotypes of dairy cattle. Can J Anim Sci, 72: 773-782.

Santoro, K.R.; Barbosa, S.B.P.; Brasil, L.H.A. e Santos, E.S. 2005. Estimativas de parâmetros de curvas de crescimento de bovinos zebu, criados no Estado de Pernambuco. Rev Bras Zootecn, 34: 2262-2279.

SAS Institute Inc. 2002. Statistical Analysis System user'sguide. Version 9.0 ed. SAS Institute. Cary. USA.

Silva, N.A.M.; Aquino, L.H.; Silva, F.F. e Oliveira, A.I.G. 2004. Curvas de crescimento e influência de fatores não-genéticos sobre as taxas de crescimento de bovinos da raça Nelore. Ciênc Agrotec, 28: 647-654.
Silva, F.L.; Alencar, M.M.; Freitas, A.R.; Packer, I.U. e Mouão, G.B. 2011 a. Curvas de crescimento em vacas de corte de diferentes tipos biológicos. Pesq Agropec Bras, 46: 262-271

Silva, N.A.M.; Lana, A.M.Q.; Silva, F.F.; Silveira, F.G.; Bergmann, M.A.; Silva, M.A. e Toral, F.L.B. 201 1b. Seleção e classificação multivariada de modelos de crescimento não lineares para bovinos Nelore. Arq Bras Med Vet Zootec, 63: 364-371.

Souza, L.A.; Caires, D.N.; Carneiro, P.L.S.; Malhado, C.H.M. e Martins Filho, R. 2010. Curvas de crescimento em bovinos da raça Indubrasil criados no Estado do Sergipe. Rev Ciênc Agron, 41: 671-676.

Tedeschi, L.O.; Boin, C.; Nardon, R.F. e Leme, P.R. 2000. Estudo da curva de crescimento de animais da raça Guzera e seus cruzamentos alimentados a pasto, com e sem suplementação. 1. Analise e seleção das funções não - lineares. Rev Bras Zootecn, 29: 630-637. 
